\title{
A New Formulation of the Diffraction Contrast Theory of Dislocations and its Application to the Weak Beam Images
}

\author{
Hwang Su Kim
}

Department of Physics, Kyungsung University, Namku, Busan 608-736, South Korea

Introduction:. The pronounced contrast asymmetry between $\pm \mathbf{g}$ weak beam diffraction images of dislocations has been frequently reported in the literature[1,2]. However these observations have not been well understood with the conventional form of the diffraction theory. For instance in the case of a scre w dislocation, the calculated images with Howie-Whelan(HW) equation give always symmetrical contrasts[3]. In this paper we report a new formulation of the theory in which the inclination of the entrance surface and the slip plane of dislocation are taken $\mathrm{i}$ nto account, which may give better understanding of the contrast asymmetry.

Formulation: In Fig. $1 \mathbf{n}_{\mathrm{s}}$ and $\mathbf{n}_{\mathrm{d}}$ are normal vectors to the entrance surface and slip planes respectively. It is assumed that the each slab of A and B is a perfect crystal but disp laced each other by $\Delta \mathbf{R}(\mathbf{r})$ due to a defect. This model is then essentially the same as multi-layered stacking fault configurations, and the formulation of the diffraction theory for this case have been done and verified experimentally by the author [4]. With slight modification of the eqn. (12) in [4], one can easily obtained the $\mathrm{N}$-beam diffracted intensity including absorption effects at the $\mathrm{E}$ in the exit surface of the column drawn in Fig. 1 as,

$$
I_{g}=\mid \sum_{m} \exp \left[\left.2 \pi i\left(\gamma_{z}^{(m)} t\right) \sum_{j} \chi_{n}^{(j, m)} C_{g}^{(j, m)}\right|^{2}\right.
$$

For the meanings of symbols in (1) and other details, one should refer to the [4]. If $\mathbf{n}_{\mathrm{s}} / / \mathbf{n}_{\mathrm{d}}$, and parallel to the beam direction, (1) can be easily manipulated to HW equation. It should be noted that in (1) the column approximation is yet employed.

Calculations: Fig. 2a,b are $+\mathbf{g}$ and $-\mathbf{g}$ diffraction intensity profiles of weak beam images $\left(\right.$ with $\mathbf{s}_{\mathrm{g}}=$ $0.1 \mathrm{~nm}^{-1}$ ) of a screw dislocation in Copper. In calculations, the thickness, $\mathrm{t}=180 \mathrm{~nm}$, the dislocation depth $y=90 \mathrm{~nm}$ and the Burger vector, $\mathbf{b}=\left[\begin{array}{lll}1 / 2 & 1 / 2 & 0\end{array}\right]$ are given. 9 beams of $\mathbf{g}=\left(\begin{array}{lll}2 & 0 & 0\end{array}\right)$ systematic row of reflections are given with the $200 \mathrm{Kv}$. The beam direction is close to $\left[\begin{array}{lll}0 & 0 & 1\end{array}\right], \quad \mathbf{n}_{\mathrm{s}}$ and $\mathbf{n}_{\mathrm{d}}$ directions are [l $\left.\begin{array}{lll}0 & 0 & 1\end{array}\right]$ and [ $\left[\begin{array}{lll}1 & -1 & 1\end{array}\right]$ respectively, which means the slip plane is $\left(\begin{array}{lll}1 & -1 & 1\end{array}\right)$ making the inclination angle, $54.7^{\circ}$ to the be am direction. In Fig. $2 \mathrm{a}, \mathrm{b}$ the dot curves are due to HW equation and clearly show contrast symmetry, but the solid curves calculated with (1) are markedly asymmetry. However this asymmetry aspect is periodically reversed roughly with increasing foil thickn ess as shown in Fig. 3, and depends on the depth, y, also. But, up to $t \quad \approx 70 \mathrm{~nm}$, no significant contrast asymmetry was seen. In this figure the degree of asymmetry (D.A.) is defined with the peak intensity as,

$$
D . A .=\left(I_{g}^{\max }-I_{-g}^{\max }\right) /\left(I_{g}^{\max }+I_{-g}^{\max }\right)
$$

Discussion: It is clear that (1) can give indeed the marked contrast asymmetry of weak beam images. Besides it is thought that even in a conventional way of contrast calculations of defects, ignoring effects of the inclination, (1) may be better manageable than the conventional form of the theory, especially when $\mathrm{N}$-beam calculations are required in such a case as of LACBED pattern simulations. 


\section{References}

[1] A.G. Cullis and G.R. Booker, Proc. $5^{\text {th }}$ Europ. Congr. Electron Microscopy, 532-533(1972).

[2] M.P. Shaw, B. Ralph and W.M. Stobbs, J. Nuclear Materials 101, 124-134 (1981).

[3] P.B. Hirsch, A. Howie, R.H. Nicholson, D.W. Pashley, and M.J. Whelan, Electron Microscopy of Thin Crystals, Krieger Publ., Huntington (N.Y.) 1977.

[4] H.S. Kim and S.S. Sh einin, phys. stat. sol. (a) 90, 231-240(1985).

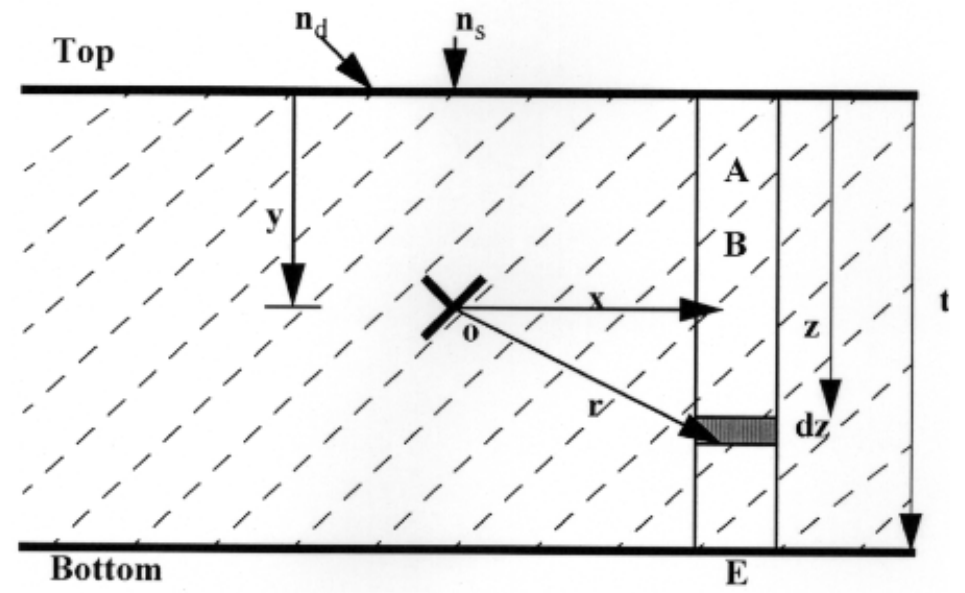

Fig. 1

Schematic diagram of a screw dislocation at the o and slip planes (dot lines), lying perpendicularly to the paper plane.

Fig. 2

a) is for the $+g$ weak beam image profiles of a screw dislocation in $\mathrm{Cu}$.

b) is for the $-\mathrm{g}$ image profiles.

The solid curves are due to eqn. (1) and the dot curves are due to HW eqn.
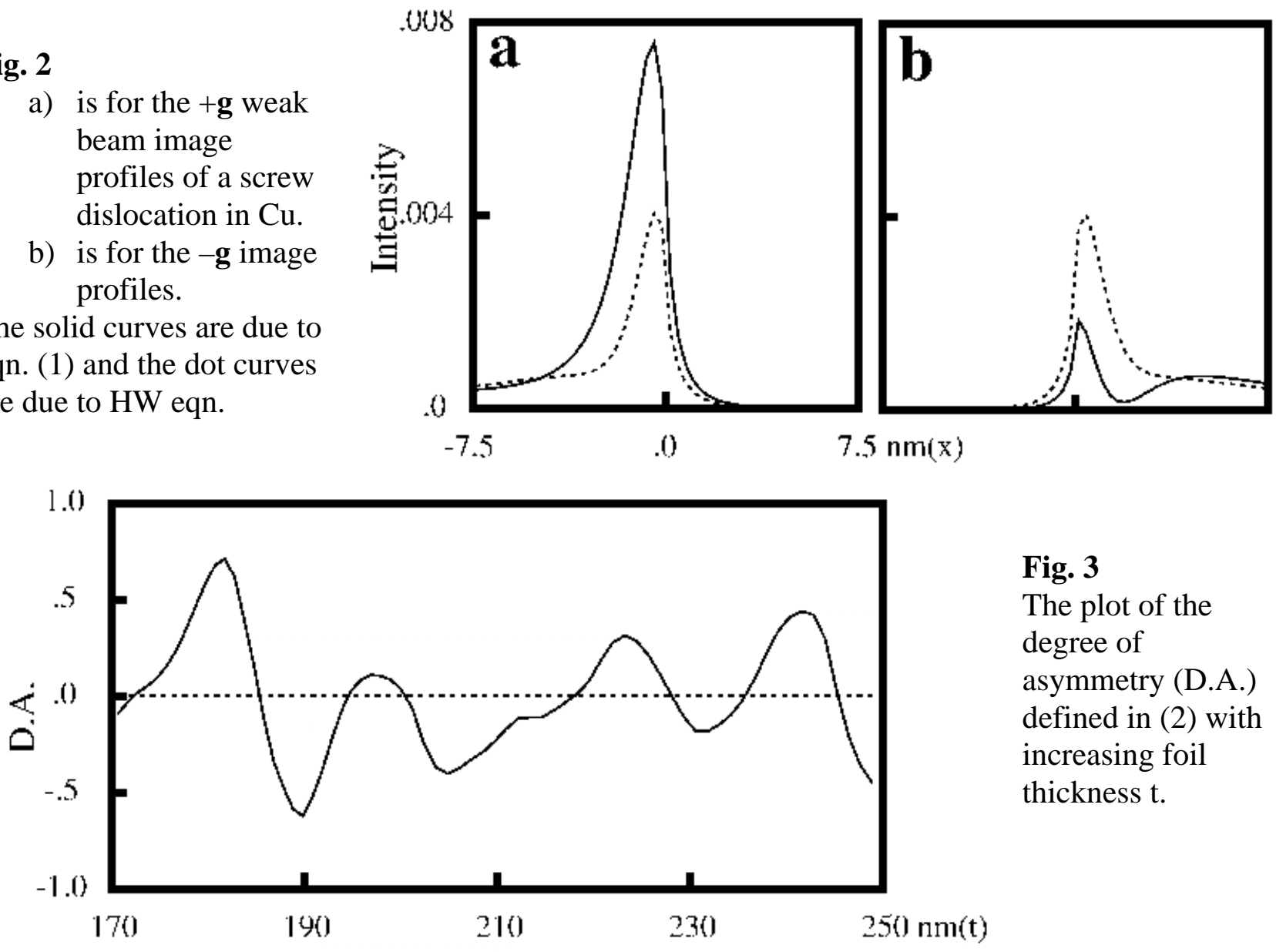

Fig. 3

The plot of the degree of asymmetry (D.A.) defined in (2) with increasing foil thickness $\mathrm{t}$. 